\title{
Tackling environmental issues in industrial ceramic sintering of sewage sludge: odors and gas emissions
}

\section{V. Cremades, $1 \square$}

Phone +34934011750

Email lazaro.cremades@upc.edu

C. Soriano, 2

J. A. Cusidó, 3

1 Department of Engineering Projects, Universitat Politècnica de Catalunya, Avda. Diagonal, 647, 08028 Barcelona, Spain

2 Centre Internacional de Mètodes Numèrics en Enginyeria (CIMNE), C/Gran Capità, s/n, Campus Nord UPC, 08034 Barcelona, Spain

3 Department of Physics and Nuclear Engineering, Universitat Politècnica de Catalunya, Pere Serra, 1-15, 08193 Barcelona, Sant Cugat del Vallès, Spain

\begin{abstract}
The inertization of sewage sludge in ceramic matrices to be used in structural or red ceramic material for buildings has proved to be a good case of reuse of waste material. However, its practical application has not yet been fully implemented in real-case scenarios, and environmental concern seems to be the main hurdle to overcome for its definitive massive approval by the building industry. In this contribution, air emissions related to the sintering of ceramic bricks made of mixtures of clay with some percentage of sewage sludge have been analyzed (in terms of gases, suspended particles and odors). Tests conducted during this work have shown higher VOC emissions in samples with some percentage of sludge in their composition (still under
\end{abstract}


the regulated emission limit values), and some of them (mercaptans) are associated with odor nuisances. Besides, limit emissions values were exceeded by three inorganic pollutants (suspended particles, $\mathrm{NO}_{\mathrm{x}}$ and $\mathrm{HCl}$ ). Measurements in an industrial scenario test showed a high variability in air pollution emissions, suggesting the need of in situ testing for definitive implementation. With the experience collected in this and several previous works in the area of emissions related to the production of added sewage sludge ceramic, some guidelines and recommendations are given to minimize the environmental impact of ceramic production plants implementing this particular waste revalorization process. Guidelines cover different aspects: workplace implementation of gas cleaning equipment; geographical context and local wind pattern analysis; monitoring of emission and immission levels; and information policy through social control participation procedures for reporting of nuisance episodes.

\section{Keywords}

Sewage sludge

Ceramic sintering

Odors

Gas emissions

Industrial production

Guidelines

\section{Introduction}

Treatment and disposal of sewage sludge resulting from wastewater treatment plant (WWTP) operation represent a high toll to pay to ensure safety of water for the population, the agriculture and the industry. Besides, the fact that this type of waste is classified as hazardous and toxic by regulations makes its pre-disposal treatment (incineration) even more costly (Han and Sculli 1991) (Fig. 1). Despite the countless number of incinerators installed in EU (Reichel et al. 2014), there is a social rejection to treating this type of waste in incineration facilities. Under this scenario, it is worth carefully analyzing any possible solution leading to a reduction in the amount of sludge to be treated. Among the different available options, valorization and reutilization are the preferred and cheapest ones. 


\section{Fig. 1}

Sewage sludge from WWTP which daily production is equivalent to $1 \mathrm{~kg}$ per inhabitant. It is a hazardous waste

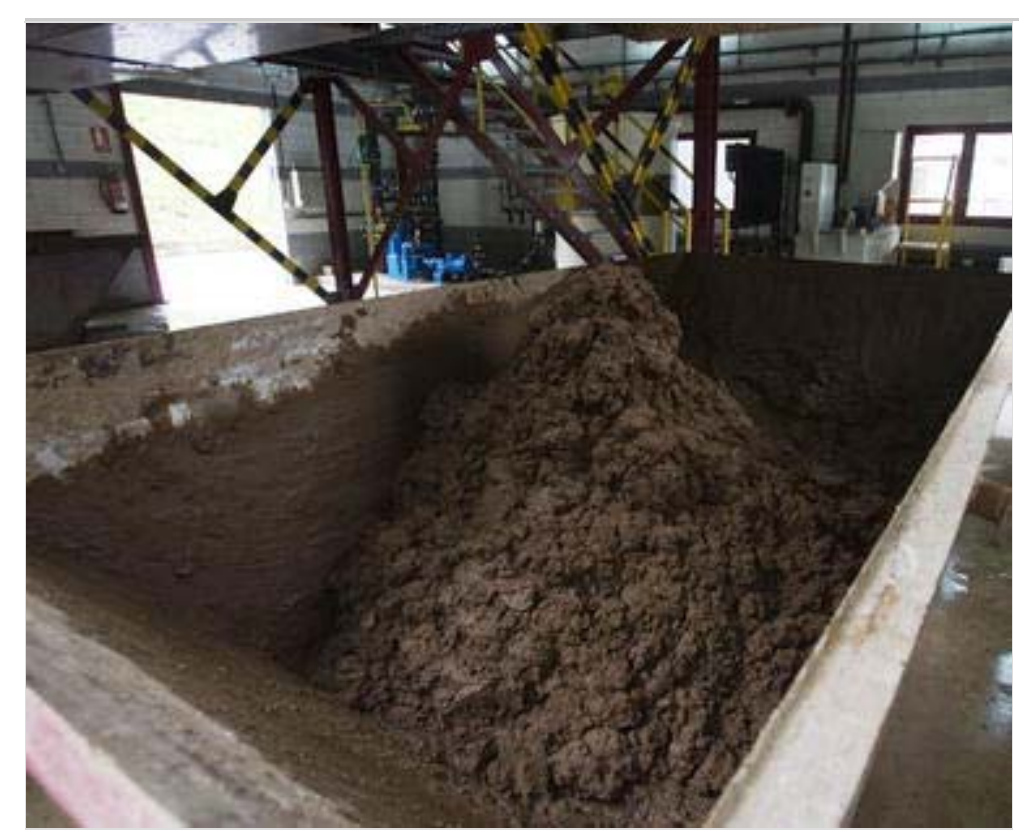

One of the possible approaches for valorization of sewage sludge is to use it as raw material for manufacturing of ceramic material for construction. This possibility has been extensively studied over the past two decades (Lin and Weng 2001; Liew et al. 2004; Jordan et al. 2005; Szoke and Muntean 2009; Chen et al. 2012; Martínez-García et al. 2014). These studies have proved the technical feasibility of ceramic material made from wet mixtures of clays with added sludge waste (in a maximum percentage of $25 \mathrm{wt} \%$ ) for its use in the construction sector.

Production of red or structural ceramics incorporating in its formulation sewage sludge from WWTP can be one of the possible ways of disposal of these toxic wastes. The thermal process destroys pathogens (viruses and bacteria), makes heavy metals inert and destroys potentially dangerous complex molecular compounds. It is necessary, however, a control and treatment of VOC produced during the process of ceramic sintering. Although this makes the production process more complex with the consequent increase in costs of implementation and maintenance, it would allow to obtain two important objectives: (1) final and secure disposal of a hazardous waste and (2) valuation in a commercial product of great demand with less weight and higher thermal 
insulation.

AQ1

Some performing properties of these new ceramic products are (Devant et al. 2011): (a) acceptable resistance to compression according to standards ( $>5 \mathrm{MPa}$ ) (UNE 67-026-94 1994), (b) significant reduction in thermal conductivity over conventional ceramic $\left(0.4 \mathrm{~W} \mathrm{~m}^{-1} \mathrm{~K}^{-1}\right.$, approximately), (c) reduction in sound transmission coefficient (51 dBA) and (d) low levels of leaching of heavy metals and other polluting compounds, below limit values in regulations (Gèric et al. 2012).

As a counterpart, gaseous emissions and odors are the main environmental issue concerning the manufacturing process of ceramic material with added sludge. However, it should be noted that the sinterization of sludge is more advantageous, in terms of air pollution, than other "waste reduction" processes such as incineration, pyrolysis, gasification and wet oxidation (Fytili and Zabaniotou 2008). In addition, with regard to $\mathrm{CO}_{2}$ emissions, sewage sludge is classified as biomass, which means that emissions related to its combustion are considered as "carbon neutral" when accounting for greenhouse gas (GHG) emission inventories and the limitations set in the Kyoto Protocol (United Nations 2015).

This paper describes first laboratory tests for characterization of raw materials and the methodology to produce sludge-added ceramic products for construction uses (clay bricks, red ceramics, etc.). Second, air pollution emissions related to the manufacturing process of this material are analyzed. Finally, some guidelines and recommendations are given for minimizing the environmental impact associated with these emissions during manufacturing of ceramic products with added sewage sludge. These guidelines will be useful for the planning of future plants or the adaptation of the existing ones.

\section{Materials and methods}

\subsection{Raw materials}

Several tests were conducted on a collection of ceramic pieces fabricated using a mixture of clays, sewage sludge from WWTP and forest debris (sawdust) at dry weight ratios of 80:15:5, respectively. The mixture was sprayed with fine droplets of water in a 10-liter mixing bowl (Controls, model BT72). Chemical composition of the major inorganic compounds of the raw materials used was obtained by X-ray fluorescence (Philips PW2510 spectrometer). Also, a qualitative analysis of the clays was done by X-ray diffractometry (Siemens XRD analyzer, model D500). Regarding heavy metal content, the samples were first calcinated at $450{ }^{\circ} \mathrm{C}$, and then, metals obtained were extracted with acid according to the EN-12457-1 standard (EN-12457-1 2002). 


\subsection{Preparation of the sludge-added ceramic samples}

To fabricate the added sludge ceramic samples, first, a mixture of clay and sludge, which will be called "clay/sludge," was dampened until getting a homogenous material. After extrusion at 10 atm (extruder Verdés, model 050-C), the collection of rectangular bars ( 5 or $12 \mathrm{~cm}$ long) were dried, first at room temperature for $24 \mathrm{~h}$ and then for another $24 \mathrm{~h}$, in a stove (Raypa, model DO-40) at $100{ }^{\circ} \mathrm{C}$. Finally, they were cooked in a propane oven (Formagas, model HG-150) up to $1000{ }^{\circ} \mathrm{C}$, by following a heating rate of $160{ }^{\circ} \mathrm{C}$ per hour. Then, a constant $1000{ }^{\circ} \mathrm{C}$ temperature was maintained in the oven for 3 more hours, following a natural cooling of the pieces at room temperature for 12 more hours (Cusidó and Soriano 2011).

\subsection{Air emissions sampling}

Gas emissions during the firing process were collected at the stack ( $300 \mathrm{~mm}$ diameter) of the oven and analyzed according to the US EPA Reference Methods (EPA, 1994). The sampling point at the stack was located at a distance from its base of $20.7 \mathrm{~m}$ above ground level (6.9 times its diameter). The distance from the top of the stack was $0.93 \mathrm{~m}$ ( 3.1 diameters). Three samples were obtained during firing one for each temperature range: $20-500{ }^{\circ} \mathrm{C}, 500-40{ }^{\circ} \mathrm{C}$ and $940{ }^{\circ} \mathrm{C}$. Calcination of sludge and sawdust takes place during the first temperature range; sintering process of clay bricks occurs during the second range; and the final ceramization of bricks is produced at $940{ }^{\circ} \mathrm{C}$ (Cusidó and Soriano 2011). It should be mentioned that test pieces with $100 \%$ clay (conventional ceramics, which we will identified with the name of "clay" sample) were also produced for the sake of comparison with mixed ceramics. The collected air samples were analyzed through gas chromatography (with mass selective detection) after using thermal desorption (Cusidó et al. 2003).

\section{Results and discussion}

\subsection{Characterization of samples}

Chemical composition of the major inorganic compounds of the raw materials used is given in Table 1. The qualitative analysis of the clays by X-ray diffractometry found that the main compounds present in the clays were: quartz $\left(\mathrm{SiO}_{2}\right)$, chlorite $\left[(\mathrm{Mg}, \mathrm{Fe})_{6}(\mathrm{SiAl})_{4} \mathrm{O}_{10}(\mathrm{OH})_{8}\right]$ and montmorillonite $\left[\mathrm{Ca}_{0.4}\left(\mathrm{Al}_{3.33} \mathrm{Mg}_{0.67}\right) \mathrm{Si}_{8} \mathrm{O}_{10}(\mathrm{OH})_{4}\right]$. Composition of organic compounds in sewage sludge was highly variable. Chemical and physical characterization of the sludge incorporated in the samples is available at Devant et al. (2011). The main heavy metals were $\mathrm{Zn}, \mathrm{Cu}$ and $\mathrm{Cr}$ with concentration values under $1000 \mathrm{ppm}$. In the case of sawdust, their main organic elements were: cellulose $(49.73 \mathrm{wt} \%)$, hemicellulose $(16.77 \mathrm{wt} \%)$ and lignine $(26.63 \mathrm{wt} \%)$. Their heavy metals 
concentrations were at the level of $1 \mathrm{ppm}$.

\section{Table 1}

Chemical composition of major inorganic compounds of the different raw materials (\% dry weight) by X-ray fluorescence

\begin{tabular}{|c|c|c|c|}
\hline & Clay & Sewage sludge & Sawdust* \\
\hline $\mathrm{SiO}_{2}$ & 51.35 & 12.36 & 0.040 \\
\hline $\mathrm{Al}_{2} \mathrm{O}_{3}$ & 17.89 & 3.25 & 0.011 \\
\hline $\mathrm{Fe}_{2} \mathrm{O}_{3}$ & 6.07 & 1.15 & 0.011 \\
\hline $\mathrm{CaO}$ & 6.53 & 7.28 & 0.087 \\
\hline $\mathrm{MgO}$ & 2.61 & 0.87 & 0.051 \\
\hline $\mathrm{Na}_{2} \mathrm{O}$ & 0.42 & 0.30 & 0.019 \\
\hline $\mathrm{K}_{2} \mathrm{O}$ & 3.61 & 0.06 & 0.072 \\
\hline $\mathrm{TiO}_{2}$ & 0.75 & & \\
\hline $\mathrm{MnO}$ & 0.09 & & \\
\hline $\mathrm{P}_{2} \mathrm{O}_{5}$ & 0.12 & & \\
\hline Total & 89.44 & 25.27 & 0.291 \\
\hline
\end{tabular}

* Ash composition after calcination at $600{ }^{\circ} \mathrm{C}$ (no organic matter)

After sintering, the resulted material had the following physical properties: apparent density, $560 \mathrm{~kg} \mathrm{~m}^{-3}$; mechanical resistance, $6.8 \mathrm{MPa}$; thermal coefficient, $0.99 \mathrm{~W} \mathrm{~m}^{-2}{ }^{\circ} \mathrm{C}^{-1}$; acoustic insulation, $51 \mathrm{dBA}$.

\subsection{Air emissions assessment related to ceramic sintering with sewage sludge}

Atmospheric emissions of volatile organic compounds (VOCs) and inorganic compounds during the firing process are included in Tables 2 and 3, respectively. The authors refer to Cusidó et al. (2003) for exhaustive details on emissions analysis. Results showed 
that VOC emissions are higher for samples including sewage sludge than for $100 \%$ clay pieces, although the threshold limit values with time-weighted average (TLV-TWA) were never exceeded for any of the cases. Only odor detection threshold values (OD) were exceeded for a few compounds (dimethyl disulfide, methyl mercaptan, acetic acid). When compared to emissions associated with direct sewage sludge burning in a one fluidized-bed incinerator, differences were observed in concentrations for complex compounds such as benzene, toluene, ethylbenzene, acrylonitrile and acetonitrile (Tirey et al. 1991). Finally, VOC emission concentrations when firing $100 \%$ clay bricks were always under the OD thresholds. For those cases, most of the VOC emissions were associated with the use of propane gas in the furnace.

\section{Table 2}

VOC emitted during the firing process ${ }^{\mathrm{a}, \mathrm{b}}$

\begin{tabular}{|c|c|c|c|c|c|c|}
\hline Family & VOC & Formula & $\begin{array}{l}\text { Clay/sludge brick } \\
\text { firing emissions } \\
\left(\mu \mathrm{g} \mathrm{m}^{-3}\right)\end{array}$ & $\begin{array}{l}\text { Clay brick firing } \\
\text { emissions }\left(\mu \mathrm{g} \mathrm{m}^{-3}\right)\end{array}$ & $\begin{array}{l}\text { TLV-TWA } \\
\left(\mu \mathrm{g} \mathrm{m}^{-3}\right)\end{array}$ & $\begin{array}{l}\mathrm{OD}^{\mathrm{d}} \\
\left(\mu \mathrm{g} \mathrm{m}^{-3}\right)\end{array}$ \\
\hline \multirow{4}{*}{$\begin{array}{l}\text { Chlorinated } \\
\text { hydrocarbons }\end{array}$} & Trichlorofluoromethane & $\mathrm{CCl}_{3} \mathrm{~F}$ & 0.0 & 571.9 & $5,620,000$ & 91,667 \\
\hline & Chloromethane & $\mathrm{CH}_{3} \mathrm{Cl}$ & 536.5 & 0.0 & 103,300 & 20,462 \\
\hline & Dichloromethane & $\mathrm{CH}_{2} \mathrm{Cl}_{2}$ & 1846.7 & 0.0 & 174,000 & 550,008 \\
\hline & Trichloromethane & $\mathrm{CHCl}_{3}$ & 179.5 & 940.5 & 48,880 & 57,184 \\
\hline Mercaptans & Methyl mercaptan & $\mathrm{CH}_{3} \mathrm{SH}$ & 16.0 & 0.0 & 980 & 1.1 \\
\hline \multirow{3}{*}{ Sulfides } & Carbon disulfide & $\mathrm{CS}_{2}$ & 728.8 & 142.7 & 31,000 & 1306.3 \\
\hline & Dimethyl disulfide & $\mathrm{S}_{2}\left(\mathrm{CH}_{3}\right)_{2}$ & 68.4 & 0.0 & 1922 & \\
\hline & Dimethyl trisulfide & $\mathrm{S}_{3}\left(\mathrm{CH}_{3}\right)_{2}$ & 0.0 & 0.0 & & \\
\hline
\end{tabular}

${ }^{\mathrm{a}}$ Units are referred to standard conditions: $T=25{ }^{\circ} \mathrm{C}$ and $P=1 \mathrm{~atm}$

${ }^{b}$ Blank values mean not available and/or not established levels

${ }^{\mathrm{c}}$ Threshold limit values with time-weighted average (ACGIH 2014)

${ }^{\mathrm{d}}$ Odour detection threshold (3M 2015) 


\begin{tabular}{|c|c|c|c|c|c|c|}
\hline Family & VOC & Formula & 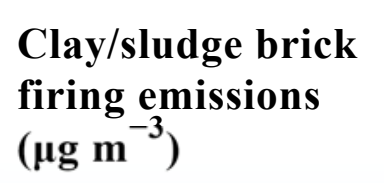 & $\begin{array}{l}\text { Clay brick firing } \\
\text { emissions }\left(\mu \mathrm{g} \mathrm{m}^{-3}\right)\end{array}$ & $\begin{array}{l}\text { TLV-TWA } \\
\left(\mu \mathrm{g} \mathrm{m}^{-3}\right)\end{array}$ & $\begin{array}{l}\mathrm{OD}^{\mathrm{d}} \\
\left(\mu \mathrm{g} \mathrm{m}^{-3}\right)\end{array}$ \\
\hline Thiocianates & Methyl thiocyanate & $\mathrm{CH}_{3} \mathrm{SCN}$ & 169.9 & 0.0 & & \\
\hline \multirow{2}{*}{ Aliphatic ketones } & Propanone & $\mathrm{C}_{3} \mathrm{H}_{6} \mathrm{O}$ & 1142.4 & 0.0 & $1,186,100$ & 147,160 \\
\hline & 3-Methyl-3-buten-2-one & $\mathrm{C}_{5} \mathrm{H}_{8} \mathrm{O}$ & 151.8 & 0.0 & 515,340 & \\
\hline \multirow{5}{*}{ Aliphatic aldehydes } & 2-Methyl propanal & $\mathrm{C}_{4} \mathrm{H}_{8} \mathrm{O}$ & 233.0 & 0.0 & 589,000 & \\
\hline & 2-Methyl propenal & $\mathrm{C}_{4} \mathrm{H}_{6} \mathrm{O}$ & 303.5 & 0.0 & & \\
\hline & 3-Methyl butanal & $\mathrm{C}_{5} \mathrm{H}_{10} \mathrm{O}$ & 305.6 & 0.0 & & \\
\hline & Hexanal & $\mathrm{C}_{6} \mathrm{H}_{12} \mathrm{O}$ & 104.7 & 0.0 & & \\
\hline & Heptanal & $\mathrm{C}_{7} \mathrm{H}_{14} \mathrm{O}$ & 183.8 & 0.0 & & \\
\hline \multirow{3}{*}{ Aromatic aldehydes } & Furfural & $\mathrm{C}_{5} \mathrm{H}_{4} \mathrm{O}_{2}$ & 255.4 & 0.0 & 7900 & 2498.6 \\
\hline & Benzoaldehyde & $\mathrm{C}_{7} \mathrm{H}_{6} \mathrm{O}$ & 594.2 & 0.0 & & \\
\hline & Hydroxybenzaldehyde & $\mathrm{C}_{7} \mathrm{H}_{6} \mathrm{O}_{2}$ & 5.3 & 0.0 & & \\
\hline \multirow{2}{*}{ Aliphatic nitriles } & Acetonitrile & $\mathrm{C}_{2} \mathrm{H}_{3} \mathrm{~N}$ & 1688.5 & 0.0 & 67,000 & 163,832 \\
\hline & Benzonitrile & $\mathrm{C}_{7} \mathrm{H}_{5} \mathrm{~N}$ & 318.5 & 0.0 & & \\
\hline \multirow{2}{*}{ Aliphatic acids } & Acetic & $\mathrm{C}_{2} \mathrm{H}_{4} \mathrm{O}_{2}$ & 2533.8 & 0.0 & 25,000 & 181.7 \\
\hline & 2-Methylpropanoic & $\mathrm{C}_{4} \mathrm{H}_{8} \mathrm{O}_{2}$ & 32.1 & 0.0 & & \\
\hline Aliphatic esters & Methyl acetate & $\mathrm{C}_{3} \mathrm{H}_{6} \mathrm{O}$ & 238.3 & 0.0 & 606,000 & 427,238 \\
\hline \multicolumn{7}{|c|}{${ }^{\mathrm{a}}$ Units are referred to standard conditions: $T=25^{\circ} \mathrm{C}$ and $P=1 \mathrm{~atm}$} \\
\hline \multicolumn{7}{|c|}{${ }^{b}$ Blank values mean not available and/or not established levels } \\
\hline \multicolumn{7}{|c|}{${ }^{\mathrm{c}}$ Threshold limit values with time-weighted average (ACGIH 2014) } \\
\hline
\end{tabular}




\begin{tabular}{|c|c|c|c|c|c|c|}
\hline Family & VOC & Formula & $\begin{array}{l}\text { Clay/sludge brick } \\
\text { firing emissions } \\
\left(\mu \mathrm{g} \mathrm{m}^{-3}\right)\end{array}$ & $\begin{array}{l}\text { Clay brick firing } \\
\text { emissions }\left(\mu \mathrm{g} \mathrm{m}^{-3}\right)\end{array}$ & $\begin{array}{l}\text { TLV-TWA } \\
\left(\mu \mathrm{g} \mathrm{m}^{-3}\right)\end{array}$ & $\begin{array}{l}\mathrm{OD}^{\mathrm{d}} \\
\left(\mu \mathrm{g} \mathrm{m}^{-3}\right)\end{array}$ \\
\hline \multirow{4}{*}{ Aromatic amines } & Pyrazine & $\mathrm{C}_{4} \mathrm{H}_{4} \mathrm{~N}_{2}$ & 160.3 & 0.0 & & \\
\hline & Pyridine & $\mathrm{C}_{5} \mathrm{H}_{5} \mathrm{~N}$ & 454.2 & 0.0 & 16,000 & 2133.7 \\
\hline & 4-Methylpyrazine & $\mathrm{C}_{5} \mathrm{H}_{6} \mathrm{~N}_{2}$ & 226.6 & 0.0 & & \\
\hline & 4-Methylpiridine & $\mathrm{C}_{6} \mathrm{H}_{7} \mathrm{~N}$ & 56.6 & 0.0 & 7610 & \\
\hline \multirow{2}{*}{ Aliphatic amides } & Acetamide & $\mathrm{C}_{2} \mathrm{H}_{5} \mathrm{NO}$ & 237.2 & 0.0 & & \\
\hline & 3-Methylbutanamide & $\mathrm{C}_{5} \mathrm{H}_{11} \mathrm{NO}$ & 0.0 & 0.0 & & \\
\hline Monoterpenes & $\alpha$-Pinene & $\mathrm{C}_{10} \mathrm{H}_{16}$ & 11.8 & 24.5 & & 64 \\
\hline \multirow{8}{*}{$\begin{array}{l}\text { Linear aliphatic } \\
\text { hydrocarbons }\end{array}$} & N-nonane & $\mathrm{C}_{9} \mathrm{H}_{20}$ & 160.3 & 0.0 & $1,050,000$ & 6596.3 \\
\hline & N-decane & $\mathrm{C}_{10} \mathrm{H}_{22}$ & 536.5 & 37.3 & & \\
\hline & N-undecane & $\mathrm{C}_{11} \mathrm{H}_{24}$ & 0.0 & 37.3 & & \\
\hline & N-dodecane & $\mathrm{C}_{12} \mathrm{H}_{26}$ & 89.8 & 0.0 & & \\
\hline & N-tridecane & $\mathrm{C}_{13} \mathrm{H}_{28}$ & 361.2 & 0.0 & & \\
\hline & N-tetradecane & $\mathrm{C}_{14} \mathrm{H}_{30}$ & 0.0 & 0.0 & & \\
\hline & $\mathrm{N}$-pentadecane & $\mathrm{C}_{15} \mathrm{H}_{32}$ & 0.0 & 0.0 & & \\
\hline & $\mathrm{N}$-hexadecane & $\mathrm{C}_{16} \mathrm{H}_{34}$ & 245.8 & 0.0 & & \\
\hline Branched aliphatic & 2,2-Dimethylpentane & $\mathrm{C}_{7} \mathrm{H}_{16}$ & 5686.4 & 10693.3 & $1,636,000$ & \\
\hline \multicolumn{7}{|c|}{${ }^{\mathrm{a}}$ Units are referred to standard conditions: $T=25^{\circ} \mathrm{C}$ and $P=1 \mathrm{~atm}$} \\
\hline \multicolumn{7}{|c|}{${ }^{b}$ Blank values mean not available and/or not established levels } \\
\hline \multicolumn{7}{|c|}{${ }^{\mathrm{c}}$ Threshold limit values with time-weighted average (ACGIH 2014) } \\
\hline
\end{tabular}




\begin{tabular}{|c|c|c|c|c|c|c|}
\hline Family & VOC & Formula & $\begin{array}{l}\text { Clay/sludge brick } \\
\text { firing emissions } \\
\left(\mu \mathrm{g} \mathrm{m}^{-3}\right)\end{array}$ & $\begin{array}{l}\text { Clay brick firing } \\
\text { emissions }\left(\mu \mathrm{g} \mathrm{m}^{-3}\right)\end{array}$ & $\begin{array}{l}\text { TLV-TWA } \\
\left(\mu \mathrm{g} \mathrm{m}^{-3}\right)\end{array}$ & $\begin{array}{l}\mathrm{OD}^{\mathrm{d}} \\
\left(\mu \mathrm{g} \mathrm{m}^{-3}\right)\end{array}$ \\
\hline \multirow{3}{*}{ hydrocarbons } & Nonanes & $\mathrm{C}_{9} \mathrm{H}_{20}$ & 0.0 & 0.0 & & \\
\hline & Decanes & $\mathrm{C}_{10} \mathrm{H}_{22}$ & 0.0 & 0.0 & & \\
\hline & Undecanes & $\mathrm{C}_{11} \mathrm{H}_{24}$ & 0.0 & 0.0 & & \\
\hline \multirow{2}{*}{$\begin{array}{l}\text { Polycyclic } \\
\text { hydrocarbons }\end{array}$} & Decahydronaphthalene & $\mathrm{C}_{12} \mathrm{H}_{18}$ & 0.0 & 0.0 & & \\
\hline & $\begin{array}{l}\text { Methyl-decahydronaph- } \\
\text { thalene }\end{array}$ & $\mathrm{C}_{13} \mathrm{H}_{20}$ & 0.0 & 0.0 & & \\
\hline \multirow{5}{*}{$\begin{array}{l}\text { Monocyclic aromatic } \\
\text { hydrocarbons }\end{array}$} & Benzene & $\mathrm{C}_{6} \mathrm{H}_{6}$ & 961.8 & 92.7 & 32,000 & 194,712 \\
\hline & Toluene & $\mathrm{C}_{7} \mathrm{H}_{8}$ & 582.4 & 182.1 & 188,000 & 6023.9 \\
\hline & Ethylbenzene & $\mathrm{C}_{8} \mathrm{H}_{10}$ & 56.6 & 0.0 & 434,000 & 2602.7 \\
\hline & $\mathrm{m}+\mathrm{p}-$ Xylene & $\mathrm{C}_{8} \mathrm{H}_{10}$ & 190.2 & 22.4 & 434,000 & 86757.2 \\
\hline & Styrene & $\mathrm{C}_{8} \mathrm{H}_{10}$ & 166.7 & 0.0 & 434,000 & 85120.3 \\
\hline \multicolumn{7}{|c|}{${ }^{\mathrm{a}}$ Units are referred to standard conditions: $T=25^{\circ} \mathrm{C}$ and $P=1 \mathrm{~atm}$} \\
\hline \multicolumn{7}{|c|}{${ }^{b}$ Blank values mean not available and/or not established levels } \\
\hline \multicolumn{7}{|c|}{${ }^{\mathrm{c}}$ Threshold limit values with time-weighted average (ACGIH 2014) } \\
\hline
\end{tabular}

\section{Table 3}

Mean emission levels of selected major inorganic compounds during the firing process and maximum limits allowed in special wastes incinerators in Catalonia ${ }^{\mathrm{a}}$

\section{Pollutant}

Particles
Units

$\mathrm{mg} \mathrm{Nm}^{-3}$

\section{Clay/sludge brick}

48.3

\section{Clay brick}

11.4
Limit allowed $^{b}$

20 


\begin{tabular}{|c|c|c|c|c|}
\hline Pollutant & Units & Clay/sludge brick & Clay brick & Limit allowed $^{b}$ \\
\hline $\mathrm{SO}_{2}$ & $\mathrm{mg} \mathrm{Nm}{ }^{-3}$ & 43 & 8.4 & 200 \\
\hline $\mathrm{CO}$ & $\mathrm{mg} \mathrm{Nm}{ }^{-3}$ & 83 & 26 & 125 \\
\hline $\mathrm{NO}_{\mathrm{x}}$ & $\mathrm{mg} \mathrm{Nm}{ }^{-3} \mathrm{NO}_{2}$ & 811 & 805 & 616 \\
\hline $\mathrm{HCl}$ & $\mathrm{mg} \mathrm{Nm}{ }^{-3}$ & 112 & 0.7 & 60 \\
\hline $\mathrm{HF}$ & $\mathrm{mg} \mathrm{Nm}{ }^{-3}$ & 1.2 & 1.6 & 4 \\
\hline $\mathrm{Cd}+\mathrm{Tl}$ & $\mathrm{mg} \mathrm{Nm}{ }^{-3}$ & 0.007 & n.d. ${ }^{\mathrm{c}}$ & 0.1 \\
\hline $\mathrm{Sb}+\mathrm{As}+\mathrm{Pb}+\mathrm{Cr}+\mathrm{Co}+\mathrm{Cu}+\mathrm{Mn}+\mathrm{Ni}+\mathrm{V}+\mathrm{Sn}$ & $\mathrm{mg} \mathrm{Nm}{ }^{-3}$ & 0.44 & 0.02 & 1 \\
\hline
\end{tabular}

${ }^{\mathrm{a}}$ Units are referred to normal conditions: $T=0{ }^{\circ} \mathrm{C}, P=1 \mathrm{~atm}, 11 \mathrm{v} \% \mathrm{O}_{2}$ and dry gas

${ }^{b}$ Decree 323/1994 of the Generalitat de Catalunya that regulates the facilities for waste incineration and determines their atmospheric emission limits. Values correspond to the strictest limits, i.e., those applicable to special waste incinerators (DOGC 1994)

${ }^{\mathrm{c}}$ Not detected

Concentrations of inorganic compounds found in the emissions during the firing process are given in Table 3 , together with the maximum limit values defined by the Catalan regulation applicable to solid waste incineration processes (DOGC 1994). Inorganic emissions during the firing process were higher for clay pieces with incorporated sludge than for $100 \%$ clay pieces (with the exception of hydrogen fluoride (HF) emission, which were higher in only-clay pieces, demonstrating that emissions for this particular compound are associated with the presence of clay in the piece, more than to the addition of waste sludge).

AQ2

Only $\mathrm{NO}_{\mathrm{x}}$, suspended particles and $\mathrm{HCl}$ showed concentrations above their respective threshold levels. Regarding $\mathrm{NO}_{\mathrm{x}}$, their emissions can be easily minimized by better control of management of the temperature and atmosphere conditions in the oven during the firing process (Patrick 1994; Niessen 2002). It is expected that during industrial implementation values below their maximum limit allowed will be found. With respect to suspended particles, their concentration is highly dependent on the oven type (streams, recirculations, etc.). Analysis listed in Table 3 showed a high $\mathrm{HCl}$ concentration in emissions in comparison to that of clay bricks. This has to do with the presence of $\mathrm{HCl}$ in the added sludge, which is associated with water chlorination during its 
waste water treatment process. However, the tendency in modern WWTP is to diminish the use of chlorine in the treatment process. This would result in a reduction in $\mathrm{HCl}$ emissions during the ceramic sintering of mixed bricks.

\section{Recommended guidelines for industrial implementation}

Implementation of any production plant of clay/sludge brick must be accompanied by specific measures to minimize the presence of pollutants in the air (emission) and the assessment of their potential area of impact in the surroundings (immission), which might affect the population directly or indirectly (through the food chain) in the medium and long term.

Since no specific air emissions regulations exist to be applied to plants leading with sewage sludge, the applicable regulations would be those regarding emission limits for urban waste incinerated promulgated by the EU (which are comparable to the limits established by the US EPA for incinerators). See Table 4. Nevertheless, the process of ceramization could be assimilated to a thermal process of pyrolysis where emissions are significantly lower than those for an incineration.

Table 4

Regulatory limits for pollutant emissions from incinerators (Batterman 2004)

\begin{tabular}{|c|c|c|c|c|c|c|c|}
\hline \multirow{2}{*}{ Pollutant } & \multirow{2}{*}{ Units } & \multicolumn{3}{|c|}{ EPA limits } & \multicolumn{3}{|l|}{ EU limits } \\
\hline & & Small & Medium & Large & Daily & Hourly & $4 \mathrm{~h}$ \\
\hline Particulate matter & $\mathrm{mg} \mathrm{dscm}^{-1}$ & 69 & 34 & 34 & 5 & 10 & \\
\hline Carbon monoxide & ppm (v) & 40 & 40 & 40 & 50 & 100 & \\
\hline \multirow{3}{*}{ Dioxins/furans } & $\mathrm{ng} \mathrm{dscm}{ }^{-1}$ total & 125 & 25 & 25 & & & \\
\hline & $\mathrm{ng} \mathrm{dscm}{ }^{-1}$ total & 2.3 & 0.6 & 0.6 & & & 0.1 \\
\hline & TEQ & & & & & & \\
\hline Organics & & & & & 5 total $\mathrm{Cl}$ & 10 & \\
\hline Hydrogen chloride & ppm (v) & 15 & 15 & 15 & 5 & 10 & \\
\hline
\end{tabular}

US EPA capacities: small $<91 \mathrm{~kg} \mathrm{~h}^{-1}$; medium $=91-227 \mathrm{~kg} \mathrm{~h}^{-1}$; large $>227 \mathrm{~kg} \mathrm{~h}^{-1}$

dscm dry standard cubic meter, $p p m(\mathrm{v})$ parts per million by volume, TEQ toxic equivalent, concentrations at $7 \mathrm{v} \% \mathrm{O}_{2}$ 


\begin{tabular}{|c|c|c|c|c|c|c|c|}
\hline \multirow{3}{*}{ Pollutant } & \multirow{2}{*}{ Units } & \multicolumn{3}{|c|}{ EPA limits } & \multicolumn{3}{|c|}{ EU limits } \\
\hline & & Small & Medium & Large & Daily & Hourly & $4 \mathrm{~h}$ \\
\hline & $\%$ reduction $(\%)$ & 99 & 99 & 99 & & & \\
\hline Sulfur dioxide & ppm (v) & 55 & 55 & 55 & 25 & 50 & \\
\hline Nitrogen oxides & ppm (v) & 250 & 250 & 250 & 100 & 200 & \\
\hline \multirow{2}{*}{ Lead } & $\mathrm{mg} \mathrm{dscm}{ }^{-1}$ & 1.2 & 0.07 & 0.07 & & & \\
\hline & $\%$ reduction (\%) & 70 & 98 & 98 & & & \\
\hline \multirow{2}{*}{ Cadmium } & $\mathrm{mg} \mathrm{dscm}^{-1}$ & 0.16 & 0.04 & 0.04 & & & 0.05 \\
\hline & $\%$ reduction $(\%)$ & 65 & 90 & 90 & & & \\
\hline \multirow{2}{*}{ Mercury } & $\mathrm{mg} \mathrm{dscm}^{-1}$ & 0.55 & 0.55 & 0.55 & & & 0.05 \\
\hline & $\%$ reduction $(\%)$ & 85 & 85 & 85 & & & \\
\hline \multicolumn{8}{|c|}{ US EPA capacities: small $<91 \mathrm{~kg} \mathrm{~h}^{-1}$; medium $=91-227 \mathrm{~kg} \mathrm{~h}^{-1} ;$ large $>227 \mathrm{~kg} \mathrm{~h}^{-1}$} \\
\hline
\end{tabular}

An industrial-scale long-term study was conducted in a ceramic plant, CEASA (Papiol, Barcelona), which was producing bricks during 10 days from $240 \mathrm{t}$ clay/sludge mixture with the same formulation as the one used in the laboratory test described above (Devant 2003) (Figs. 2 and 3). Its emissions contained high levels of CO (1100 mg Nm $\left.{ }^{-3}\right)$, TOC $\left(1085 \mathrm{mg} \mathrm{Nm}^{-3}\right)$ and $\mathrm{HCl}$ $\left(71 \mathrm{mg} \mathrm{Nm}^{-3}\right)$. These levels are above the regulated emission limits. It was detected the existence of unburnt organic matter which was responsible for strong smells. $\mathrm{HCl}$ emission was associated with the presence of chlorine in both, clays and sewage sludge, which can be corrected by adapting the furnace firing curve and filters. From the analysis of the emitted gases, a total of 57 VOC were found. The most significant compounds were: benzene $\left(\mathrm{C}_{6} \mathrm{H}_{6}, 1172 \mathrm{mg} \mathrm{Nm}^{-3}\right)$, dimethyl disulfide $\left(\mathrm{C}_{2} \mathrm{H}_{6} \mathrm{~S}_{2}, 1383 \mathrm{mg} \mathrm{Nm}{ }^{-3}\right)$, toluene $\left(\mathrm{C}_{7} \mathrm{H}_{8}, 926 \mathrm{mg} \mathrm{Nm}^{-3}\right)$, 4-methylpentanenitrile $\left(\mathrm{C}_{6} \mathrm{H}_{11} \mathrm{~N}, 697 \mathrm{mg} \mathrm{Nm}^{-3}\right)$, benzonitrile + isociane-benzene $\left(\mathrm{C}_{7} \mathrm{H}_{5} \mathrm{~N}+\mathrm{C}_{7} \mathrm{H}_{5}\right.$, $\left.1003 \mathrm{mg} \mathrm{Nm}^{-3}\right)$.

Fig. 2 


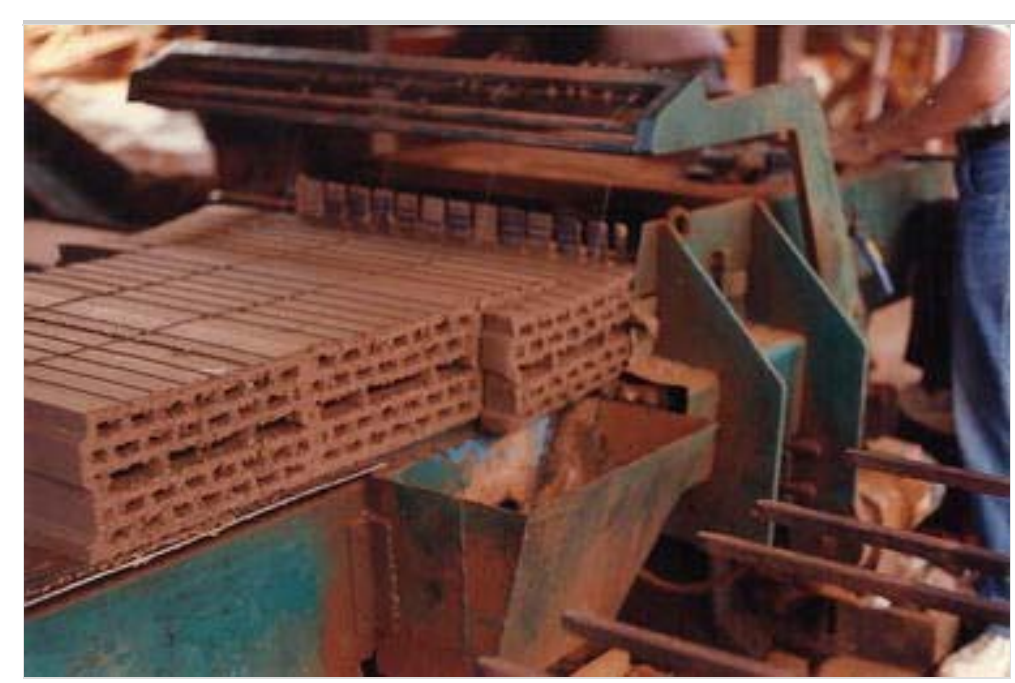

\section{Fig. 3}

Picture of the texture of the clay/sludge material after sintering, showing great porosity due to the organic matter present in initial composition. This gives interesting thermal and acoustic properties to the final product (Cusidó et al. 2003)

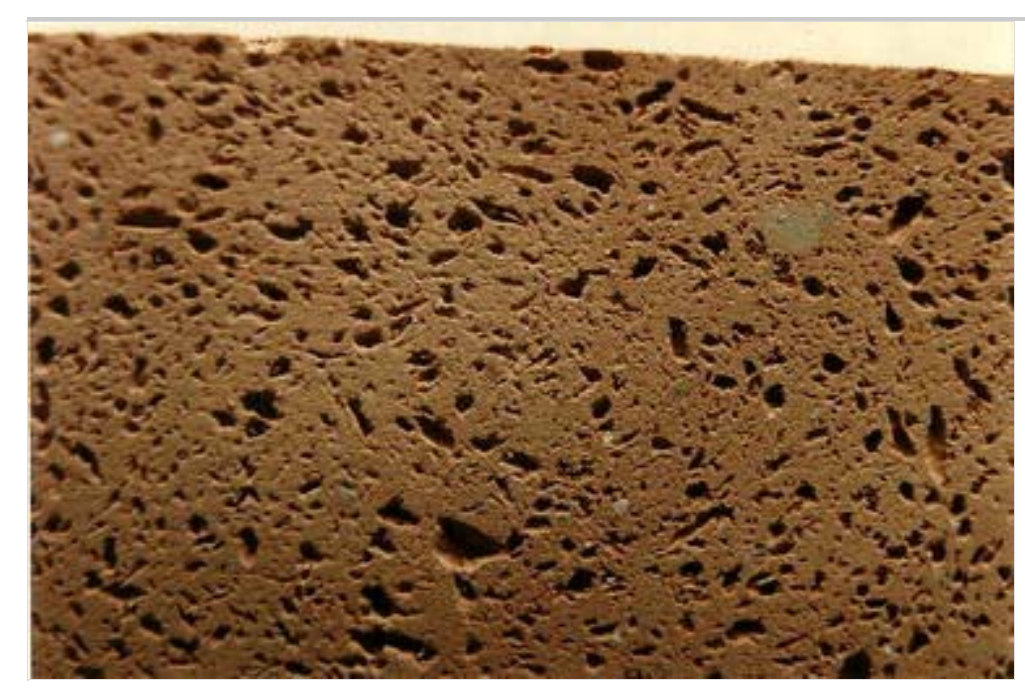

These results suggest that there is a high variability in air pollution emissions related to firing clay/sludge and are highly dependent 
on the firing curve and the furnace conditions. Therefore, it is always advisable to perform not only previous laboratory-scale studies of gaseous emissions and odors, but also in situ emissions studies in the industrial plant to assess the degree of compliance of emissions regulations.

Regarding immissions, study of the dispersion of gases, particles and odors mathematical models for continuous point sources can be applied, being the Gaussian plume model a well-known option (Stockie 2011). There are a number of available computer programs based on this scheme, such as some of the models recommended by the Support Center for Regulatory Atmospheric Modeling of the US EPA [i.e., AERMOD or CALPUFF (SCRAM 2015)]. 3D mesoscale atmospheric and dispersion models are able to predict the behavior and evolution of relevant variables (wind speed and direction, pressure, temperature, humidity content and precipitation). These types of models use numerical methods to solve equations representing a fluid in a rotational system (air) and its interactions with the terrain. They also can reproduce the different energy exchanges during the daily cycle. In turn, they require more computing power than the Gaussian ones. An example of this type of modeling is TAPM (The Air Pollution Model) (Hurley 2005).

From all the information presented, some recommendations and guidelines are suggested to be taken into account to minimize the impact on air quality associated with the manufacturing of this sludge-added material:

(i) Implementations related to the workplace:

- Use of a tower for biological washing of gases that are introduced into the kiln.

- Cyclones to capture particulate emissions from stacks,

- Post-combustion cycle of at least $2 \mathrm{~s}$ of residence time between 800 and $900{ }^{\circ} \mathrm{C}$ and $20 \%$ excess air to complete combustion of VOC, dioxins, furans and CO (Niessen 2002).

- A system of gas/gas exchanger is also recommended for the utilization of gases for drying or pre-baking (e.g., Solvay NEUTREC ${ }^{\circledR}$ system).

- Biological filters or other methods for removing odors (if the site is close to residential areas).

- Applying all other considerations regarding the treatment of toxic and hazardous wastes as described by EPA (1990) and 
subsequent regulations.

(ii) Regarding the location of the plant (either new or adapted from a preexisting one):

- Recommended locations would be in rural and sparsely populated environments.

- Knowledge of the climatic characteristics of the site (solar radiation, humidity and, in particular, the existence of weather situations of temperature inversion of great importance in the selection of the location).

- Good knowledge of local wind patterns including an orographic study. Wind causes a horizontal dispersion of air pollutants and determines the area that will be exposed to immission. Generally, the higher the wind speed, the lower the concentrations of pollutants at ground level since there would be a greater dispersion and mixing. However, adverse weather conditions (closed wind circulations, such as sea breezes, or temperature inversions) can create negative conditions by increasing pollutant concentrations in certain areas due to convective vertical transport.

(iii) Control of emissions/immissions:

- Implementation of air quality monitoring plans for air emission parameters (concentrations, flow rates, temperatures).

- Regarding immission concentrations, monitoring of the impacted area requires the installation of sensors for the following parameters: (a) continuous emission monitoring (CEM) of particles, $\mathrm{SO}_{2}, \mathrm{CO}, \mathrm{O}_{2}, \mathrm{NO}_{\mathrm{x}}, \mathrm{HCl}$ among others depending on each case, (b) on a regular basis or even exceptionally in our case, stack tests of dioxins, furans, heavy metals, being unnecessary measurements of $\mathrm{Hg}$, (c) environmental monitoring (air, water, soil, food, etc.) around the industrial plant in a random manner.

- It is recommended to facilitate inspections, certifications of qualification of the personnel as well as sanitary control, safety equipment, etc.

(iv) Information policy:

- This type of installations is usually associated with the existence of a strong social opposition to the implementation of 
the process of sludge inertization in ceramic matrices. It often creates social alarm due to unfamiliarity and lack of information about this novel process. Establishing a line of serious and truthful information can help achieve the acquiescence of the surrounding population, in particular if they are thoroughly informed about the implementation of measures of environmental prevention and monitoring in order to minimize/reduce risk to the health of the inhabitants.

- On the other hand, to inform population on the environmental benefits of the technology (reduction in GHG and waste in landfills) and the products obtained should contribute to the acceptance of the definitive industrial implementation of the new ceramic product.

It is advisable to implement social control participation procedures for engaging residents in the notification and reporting of nuisance episodes on a regular basis, particularly related to odor episodes (Gallego et al. 2008).

\section{Conclusions}

The production of ceramic material for construction from clay/sewage sludge mixtures can be a real solution for the safe disposal of waste from WWTP. However, environmental issues seem to be an obstacle to its widespread implementation, despite its several advantages (economically and environmentally).

Tests carried out on ceramic pieces obtained using clay/sludge found that particles and $\mathrm{HCl}$ are the compounds whose concentrations exceed the permitted limit values. Regarding the $\mathrm{NO}_{\mathrm{x}}$ emissions, they also exceed their limit values but emission concentrations of the same order as in conventional production of clay brick. The only organic compound that far exceeded its OD was methyl mercaptan.

When compared to incineration of sludge and its associated high load of air pollution emissions, the option of valorization of sludge as raw material seems to be a good alternative for production of ceramic products of high demand (such as clay bricks).

For the implementation of the industrial production, it is advisable to carry out laboratory studies, since these residues (as well as the clays) have very varying characteristics depending of their site of origin. The application of the regulations of each country, as well as the existence of technologies for the treatment of gases, odors and particulates, would allow a clean production in accordance with legal standards. 
Some guidelines have been proposed to ensure the industrial operation of a new industrial production line, under minimum impact on the surrounding area. If well designed, emissions from this process are lower than those of any other currently available process for thermal treatment of sewage waste. In addition, this production implies a complete removal of sludge as a waste and its valorization into a commercial product with added value.

\section{Acknowledgements}

This work stems from the experience gained by the authors in the development of the project BRE2.CT94.1575 that was financed by the European Commission (BRITE-Euram; CRAFT Programme).

\section{References}

3M (2015). 3 M Respirator Selection Guide. URL: https://multimedia.3m.com/mws/media/639110O/3m-respirator-selectionguide.pdf (Accessed 2 February 2017).

ACGIH (2014). Threshold limit values and biological exposure indices. American Conference of Governmental Industrial Hygienists. ACGIH Signature Publications.

Batterman, S. (2004). Small-scale incinerators for health-care waste. WHO. URL: http://apps.who.int/iris/bitstream/10665 168775/1/a85187.pdf (Accessed 8 May 2015).

Chen, H. J., Yang, M. D., Tang, C. W., \& Wang, S. Y. (2012). Producing synthetic lightweight aggregates from reservoir sediments. Construction and Building Materials, 28(1), 387-394.

Cusidó, J. A., Cremades, L. V., \& González, M. (2003). Gaseous emissions from ceramic manufactured with urban sewage sludges during firing processes. Waste Management, 23, 273-280.

Cusidó, J. A., \& Soriano, C. (2011). Valorization of pellets from municipal WWTP sludge in lightweight clay ceramics. Waste Management, 31, 1372-1380. 
Devant, M. (2003). Resultats de la prova industrial Ecobrick i les possibles millores industrials (Results of the industrial test Ecobrick and possible industrial improvements). Technical report for CEASA, Barcelona [in Catalan].

Devant, M., Cusidó, J. A., \& Soriano, C. (2011). Custom formulation of red ceramics with clay, sewage sludge and forest waste. Applied Clay Science, 54, 669-675.

DOGC (Diari Oficial de la Generalitat de Catalunya). (1994). Decree 323/1994 regulating the installations of waste incineration and their air emission limits. Department of Environment (Catalonian Government). Oficial Bulletin of the Generalitat de Catalunya, 1986, 8200-8205. [in Catalan].

EN-12457-1 (2002). Characterization of waste. Leaching: Compliance test for leaching of granular waste materials and sludges. UE. Publication date 15.10.2002.

EPA (U.S. Environmental Protection Agency). (1990). RCRA orientation manual. Washington D.C.: Office of Solid Waste, Environmental Protection Agency.

EPA (U.S. Environmental Protection Agency)(1994).New Source Performance Standards (NSPS). Code of Federal Regulations, 40 CRF 60, Washington D.C.

Fytili, D., \& Zabaniotou, A. (2008). Utilization of sewage sludge in EU application of old and new methods-A review. Renewable and Sustainable Energ. Reviews, 12, 116-140.

Gallego, E., Soriano, C., Roca, F. X., \& Guardino, X. (2008). Identification of the origin of odour episodes through social participation, chemical control and numerical modelling. Atmospheric Environment, 42(35), 8150-8160.

Gèric, M., Gajski, G., Orescanin, V., Kollar, R., \& Garaj-Vrhovac, V. (2012). Chemical and toxicological characteristics of the bricks produced from clay/sewage sludge mixture. Journal of Environmental Science and Health A, 47, 1521-1527.

Han, K. C., \& Sculli, D. (1991). Cost of disposal of sewage sludge. Engineering Costs and Production Economics, 21, $133-141$. 
Hurley, P.J. (2005). The air pollution model (TAPM) Version 3. Part1, “Technical description”, CSIRO Atmospheric Research, Tehnical Paper No.71, Australia.

Jordan, M. M., Almendro-Candel, M. B., Romero, M., \& Rincon, J. M. (2005). Application of sewage sludges in the manufacturing of ceramic tile bodies. Applied Clay Science, 30, 219-224.

Liew, A. G., Idris, A., Samad, A. A., Wong, C. H. K., Jaafar, M. S., \& Baki, A. M. (2004). Reusability of sewage sludge in clay bricks. Journal of Material Cycles and Waste Management, 6, 41-47.

Lin, D.-F., \& Weng, C.-H. (2001). Use of sewage sludge ash as a brick material. Journal of Environmental Engineering, 127, 922-927.

Martínez-García, C., Cotes Palomino, M. T., Iglesias-Godino, F. J., \& Corpas-Iglesias, F. A. (2014). Porosity of expanded clay manufactured with addition of sludge from the brewing industry. International Journal of Energy and Environmental Engineering, 5(4), 341-347.

Niessen, W. R. (2002). Combustion and incineration processes, applications in environmental engineering (3rd ed.). New York: Marcel Dekker Inc.

Patrick, D. R. (1994). Toxic air pollution handbook (1st ed.). New York: Van Nostrand Reinhold.

Reichel, A., Wilts, H., Von Gries, N. (2014). Municipal solid waste management capacities in Europe. ETC/SCP Working Paper No. 8/2014. URL: http://scp.eionet.europa.eu/publications/wp2014_8/wp/wp2014_8 (Accessed 8 May 2015).

SCRAM (Support Center for Regulatory Atmospheric Modeling) (2015). Preferred/recomended models. U.S. EPA. URL:http://www.epa.gov/ttn/scram/dispersion_prefrec.htm (Accessed 8 May 2015).

Stockie, J. M. (2011). The mathematics of atmospheric dispersion modeling. SIAM Review, 53, 349-372.

Szoke, A. M., \& Muntean, M. (2009). Sludge recycling in ceramic matrix. Environmental Engineering and Management 
Journal (EEMJ), 8, 907-909.

Tirey, D. A., Striebich, R. C., Dellinger, B., \& Bostian, H. E. (1991). Comparison of organic emissions from laboratory and full-scale thermal degradation of sewage sludge. Hazardous Waste and Hazardous Materials, 8, 201-218.

UNE 67-026-94 (1994). Bricks and ceramic blocks made of clay. Determination of compressive strength [in Spanish].

United Nations (2015). Kyoto Protocol. URL: http://unfccc.int/kyoto_protocol/items/2830.php (Accessed 13 May 2015). 